Learning to Think in a Second Language: Effects of Proficiency and Length of Exposure in

English Learners of German

\title{
PANOS ATHANASOPOULOS
}

Lancaster University

Department of Linguistics and English Language

County South

Bailrigg

Lancaster

LA1 4YL

United Kingdom

p.athanasopoulos@lancaster.ac.uk

University of Reading

Centre for Literacy and Multilingualism

School of Psychology and Clinieal Language Seiences

University of Reading

Reading RG6 6AL

United Kingdom

p.athanasopoulos@reading.ae.uk

LJUBICA DAMJANOVIC

University of Chester

Department of Psychology

Parkgate Road

Chester

CH1 4BJ

United Kingdom

1.damjanovic@.chester.ac.uk

JULIE BURNAND

University of Chester

Department of Psychology

Parkgate Road

Chester

CH1 4BJ

United Kingdom

julieburnand@.gmail.com

EMANUEL BYLUND

Stockholm University \& Stellenbosch University

Centre for Research on Bilingualism

Department of Swedish and Multilingualism

Universitetsvägen 10C 
10691 Stockholm

Sweden

manne.bylund@,biling.su.se

$<$ ABSTRACT $>$

The aim of the current study is to investigate motion event cognition in second language learners in a higher education context. Based on recent findings that speakers of grammatical aspect languages like English attend less to the endpoint (goal) of events than do speakers of non-aspect languages like Swedish in a nonverbal categorization task involving working memory (Athanasopoulos \& Bylund, 2013; Bylund \& Athanasopoulos, 2015), the current study asks whether native speakers of an aspect language start paying more attention to event endpoints when learning a non-aspect language. Native English and German (a non-aspect language) speakers, and English learners of L2 German, who were pursuing studies in German language and literature at an English university, were asked to match a target scene with intermediate degree of endpoint orientation with two alternate scenes with low and high degree of endpoint orientation, respectively. Results showed that, compared to the native English speakers, the learners of German were more prone to base their similarity judgements on endpoint saliency, rather than ongoingness, primarily as a function of increasing L2 proficiency and year of university study. Further analyses revealed a non-linear relationship between length of L2 exposure and categorization patterns, subserved by a progressive strengthening of the relationship between L2 proficiency and categorization as length of exposure increased. These findings present evidence that cognitive restructuring may occur through increasing experience with an L2, but also suggest that this relationship may be complex and unfold over a long period of time. 
$<$ END ABSTRACT $>$

Keywords: German as a foreign language; grammatical aspect; boundedness; linguistic relativity; instructed SLA; motion events

The idea that the native language can affect thinking and reasoning about the perceived world has a long history in scholarly thought. In modern cognitive science, this idea is often referred to as the linguistic relativity hypothesis (Whorf, 1956). While the hypothesis has evolved and diversified into more fine-grained proposals (see Wolff \& Holmes, 2010, for a detailed overview), the basic principle is that crosslinguistic differences in the semantic partitioning of reality (language diversity) may give rise to crosslinguistic differences in thought, the latter typically operationalized as non-verbal behaviour related to a range of different cognitive processes, such as reasoning, classification, and categorical perception (Lucy, 1997). Recent studies show effects of linguistic categories on cognitive processes in a variety of domains, such as colour (Regier \& Kay, 2009), grammatical number (Lucy \& Gaskins, 2003), grammatical gender (Boutonnet, Athanasopoulos, \& Thierry, 2012), space (Majid et al., 2004), time (Casasanto, 2008), and manner and path of motion (Kersten et al., 2010). While the extent of linguistic influence may vary as a function of domain and task and while the reported effects do not go uncontested (see e.g., Barner, Li, \& Inagaki, 2009; January \& Kako, 2007; Li \& Gleitman, 2002), the linguistic relativity hypothesis forms a core part of the investigation into human development and behaviour throughout the cognitive and social sciences.

Given the substantial cross-linguistic diversity that exists in how languages semantically partition reality and the correlation of this diversity with different cognitive patterns in monolingual populations, recent studies have investigated the linguistic relativity 
hypothesis through the lens of bilingualism. The main question here takes as its starting point cognitive differences in monolingual populations in a specific domain and examines the extent to which language-specific cognition in the same domain is resistant to L2 influence, or whether cognitive patterns may change under the influence of the L2 (Jarvis \& Pavlenko, 2008). Studies to date have shown that cognitive categorisation may be impervious to L2 influence in intermediate L2 users (Athanasopoulos, 2006) or late bilinguals (Boroditsky, Schmidt, \& Phillips, 2003), but may be influenced by the L2 once an advanced level of proficiency has been reached (Athanasopoulos \& Kasai, 2008), in early bilinguals (Boroditsky, 2001), and as a function of language mode (Kersten et al., 2010).

The current study is located within the grammatical aspect approach to motion event cognition (von Stutterheim et al., 2012). More specifically, it builds on recent evidence that native speakers of languages like English that obligatorily mark progressive aspect to express ongoingness (e.g., 'John is walking') attend less to the endpoint (goal) of an action than do speakers of non-aspect languages like Swedish (where ongoingness can be optionally expressed outside of the main verb through adverbials or other lexical means) in a nonverbal similarity judgement task involving working memory (Athanasopoulos \& Bylund, 2013). Existing data provide some evidence that functional multilingual speakers whose L1 lacks grammatical aspect (and thus promotes attention to endpoints) but whose L2 marks progressive aspect grammatically (and thus defocuses endpoints) are influenced by their L2 in their event categorization behaviour as a function of frequency of L2 use and exposure to the L2 as the language of schooling in childhood (Bylund et al., 2013; Bylund \& Athanasopoulos, 2014). It seems that going from an L1 that lacks grammaticized means to express progressive aspect to an L2 where such means are applied obligatorily may have profound consequences for event cognition. 
This article contributes to this line of investigation in two ways. First, we explore whether cognitive restructuring is possible when going in the other direction, that is, when the $\mathrm{L} 1$ is a [+ aspect] language and the $\mathrm{L} 2$ is a [- aspect] language. One may assume that cognitive restructuring occurs in the direction of [- aspect $]$ L1 to [+ aspect $]$ L2 as a function of the acquisition of a new, obligatorily applied grammatical category (in this case, progressive aspect). However, going in the opposite direction, there are no obligatory grammatical categories to be acquired. Therefore one could assume that cognitive restructuring may not occur at all, since obligatoriness of application of a grammatical category may be a key factor in the extent of linguistic influence on cognition (Lucy, 1992). Second, we examine the idea that linguistic relativity effects emerge in humans primarily as a function of degree of exposure to a specific language. Recent attempts to identify the theoretical underpinnings of cognitive restructuring in L2 users have adopted models of associative learning (see subsequent sections) that assume that changes in cognition emerge gradually as a function of the degree of exposure to specific form-meaning associations, which then become part of an individual's cognitive routine (Bylund \& Athanasopoulos, 2015; Casasanto, 2008; Kersten et al., 2010). If that is so, the longer learners are exposed to a second language with grammatical properties different from the first, the more their cognitive dispositions are likely to shift towards those of native speakers of the L2. Thus the current study focuses on length of L2 exposure in additional language learners who are not immersed in the target language context and on the evolution of relationships between other biographical variables and event cognition as such length unfolds.

\footnotetext{
$<$ A $>$ BACKGROUND

$<B>$ Theoretical Approach
} 
The current study is located within the theoretical framework of Cognitive Linguistics (CL) (see Evans, Bergen, \& Zinken, 2007; Geeraertz \& Cuyckens, 2007, for detailed insightful introductions), which places the task of language learning within general principles of human cognitive processing. It views language not as an autonomous modular system with its own rules and completely unrelated to other cognitive processes, but as an integrative component of general human cognitive processing. As such, it resonates with linguistic relativity and thinking-for-speaking approaches to L2 learning (Pavlenko, 2011), and lends importance to non-verbal behaviour alongside verbal behaviour in describing phenomena of language learning, representation, and use.

Three central concepts or key insights from a Cognitive Linguistic approach to L2 learning are relevant for the present investigation, namely construal, categorization, and the usage-based nature of language. The first two refer to the basic cognitive operations at play here. The third refers to the learning mechanism to which these operations are amenable.

Construal refers to the ability to take different perspectives or interpretations of an event or, for that matter, any perceptual phenomenon (Langacker, 1987, 1991). In his discussion on the similarity between lexical and grammatical categories, Langacker (2008) postulates that grammatical constructions are form-meaning pairings above the word level, with cross-linguistic differences in grammar essentially referring to different conventionalized linguistic constructions for encoding different construals of the same external event. A change in grammatical or syntactic form entails a change in meaning, which in turn entails a shift in construal. Grammatical aspect, the focus of the current study, is a primary linguistic marker of different temporal construals. Imagine a scene where two men are walking along a path, at the end of which there is a bridge. Before the men reach the bridge, the scene ends. An observer who is asked to describe this scene may choose to adopt a holistic perspective, or a maximal temporal viewing frame, according to which the event is 
interpreted in its entirety, including the endpoint (i.e., two men walking to a bridge). The observer, however, could also adopt an alternative interpretation, or construal, choosing that of an immediate temporal viewing frame, whereby only the ongoing phase of the event is zoomed in on and the event endpoint is excluded (i.e., two men walking) (Langacker 1987, 2008). A growing number of studies have shown that the choice of event construal (holistic or immediate) is tightly linked to the presence or absence of the grammatical category of aspect in the observer's/speaker's native language. Evidence from speech production data demonstrates that when describing unfolding goal-oriented motion events, speakers of languages with grammatical aspect are more prone to adopting an immediate viewing frame and exclude reference to endpoints. Speakers of languages lacking grammatical aspect, in contrast, show a higher preference to take a maximal viewing frame, mentioning the event endpoint. This finding has been shown to be consistent across a number of different languages, such as Algerian Arabic, English, Russian, Spanish (aspect languages), and Dutch, German, Norwegian, Swedish (non-aspect languages) (Bylund, 2011; Schmiedtová \& Flecken, 2008; von Stutterheim, 1997, 2003).

Varying the construal of the same event may have immediate consequences for the second cognitive process mentioned earlier, namely categorization. Generally speaking, categorization is an essential element of human cognition. Indeed, under many cognitive linguistic and psychological accounts cognition is essentially categorization (Harnad, 1987, 2005; Roberson, 2005). The central operating principle of this process is that similarity is the basis of categorisation (Ameel et al., 2005; Nosofsky, 1986), a position that constitutes the basic methodological tenet of modern neo-Whorfian investigations, where non-verbal behaviour is often operationalised along a continuum of cognitive tasks with an inherent categorisation component (Casasanto, 2008; Lucy, 1997). A classic example of this is the triads matching task, in which participants are asked to match a target stimulus with one out 
of two alternate stimuli. Among other domains, such as object categorization (Lucy, 1992) and colour categorical perception (Roberson, 2005), the triads matching paradigm has also been successfully implemented in the domain of grammatical aspect and event construal. The basic assumption is that if two events are construed (interpreted) similarly, then they will tend to be perceived and categorized as more similar in a triads matching task.

Athanasopoulos and Bylund (2013) examined the influence of grammatical aspect on non-verbal event cognition in a triads matching design, asking participants to watch triads of video clips showing motion events with different degrees of goal orientation. The target clip showed motion towards a goal (intermediate degree of goal orientation), whereas the alternates showed either motion without an obvious endpoint (low degree of goal orientation) or motion with arrival at a goal (high degree of goal orientation). The clips were presented in three different conditions: one where the clips in each triad were played simultaneously in a loop until the participant had made his/her judgment ("online condition"); one where the clips played one after another and the participant provided his/her judgment after having watched the last clip in each triad ("memory condition"); and one similar to the memory condition with the exception that the participant had to repeat a string of digits while watching each triad ("verbal interference condition").

The results showed that in the memory condition speakers of Swedish paired the target clip with the high degree alternate significantly more than English speakers did. No cognitive differences between the two populations of speakers were found in the other conditions. Athanasopoulos and Bylund (2013) argued that the reason why differences between groups were found in the memory condition was due to the fact that working memory is, to some extent, verbally mediated (Baddeley, 2003). These results thus suggest that the effects of grammatical aspect on non-verbal event cognition are primarily confined to situations where the speaker has to commit facts to memory and is able to rely on language to 
do so. Such non-verbal behaviour open to verbal mediation probably forms the majority of real-life instances of categorization and higher level problem solving in humans (Kersten et al., 2010).

How do such effects play out in instances of multilingualism and additional language learning? To begin to answer this question, we turn to the third key insight from a Cognitive Linguistic account of L2 learning, namely the usage-based nature of language that is anchored to more general principles of associative learning. Over the past 10 years or so, the psychological theory of associative learning has gained some prominence in L1 development accounts (Colunga \& Smith, 2005; Samuelson, 2002; Smith, 2000; 2003), in L2 acquisition research (Ellis, 1998; 2002; 2006) and in Cognitive Linguistic theory of language (Bybee, 2006; Ellis \& Ferreira-Junior, 2009). More recently, it has been proposed as a theoretical framework to explain linguistic relativity effects (Casasanto, 2008; Kersten et al., 2010) and cognitive restructuring in L2 acquisition/multilingualism (Bylund \& Athanasopoulos, 2015). The starting point in linking associative learning to cognitive restructuring in L2 is the observation that representations in memory are multi-modal and massively interconnected. Thus, when participants hear verbs like 'kick' and 'grasp', and when participants actually kick or grasp something, neuroscientific evidence shows that the same neural assemblies are activated (Kiefer \& Pulvermüller, 2012). Processing of colour words activates both the expected language areas as well as areas in the visual cortex associated with colour vision (Regier \& Kay, 2009). Representations then build up, or emerge, over exposure to a number of specific instances of associations. For example, speakers of English will form distinct form-meaning representations for blue and green, whereas speakers of Berinmo and Himba (whose languages have only one term for blue and green) will associate only one term with the same physical space of the colour spectrum, because during L1 development two distinct terms will statistically co-occur with two different visual representations in the input English 
children are exposed to, whereas for Berinmo and Himba children only one term will frequently co-occur with visual representations of colour space that English speakers would normally distinguish into 'green' and 'blue' (Roberson et al., 2004).

Linguistic relativity effects arise because we draw on co-occurrence patterns that we have encountered when we construe and categorize a specific event, and when we encounter a new event. Regularities in categorization emerge as a function of the degree of exposure to specific associations, which then become part of an individual's cognitive routine. The more routinized an association becomes, the easier it is to retrieve and utilize it for purposes of categorization (Langacker, 2008). Indeed, the evidence shows that the emergence of crosslinguistic differences in colour categorization is a graded, linear process, occurring as soon as children show signs of acquisition of their language-specific colour lexicons, and increasing with age (Franklin et al., 2008; Roberson et al., 2004). Similar developmental trends have been reported for categorization of objects and substances (Imai \& Gentner, 1997; Lucy \& Gaskins, 2001). At the same time, because we are continuously exposed to novel events throughout our lifetime representation is subject to constant restructuring. The individual constantly updates the relative statistical weighting of the recurring associations against new contexts and new instances of associations (Ellis, 2008). The question of interest from an L2 learning perspective then concerns the extent to which construal and categorization are affected by the acquisition of novel form-meaning associations in the L2, and the factors that modulate internalization of these associations.

From an associative learning perspective, the key factor is increasing experience with specific form-meaning associations. However, the construct of 'learning experience' itself is multifaceted, and must be operationalized in light of the learning context. For example, in L1 acquisition, experience is instantiated as repeated exposure to the ambient language environment pre- and post-natally, which has specific consequences for how infants 
understand phonemic features of the ambient language and begin segmenting the input based on statistical regularities (Johnson \& Jusczyk, 2001) and for how they construct morphophonological representations (Szagun, 2001). Frequency of exposure to specific word classes has consequences for categorization of objects and events (Gopnik, Choi, \& Baumberger, 1996). Arguably, for L2 learners, the construct of experience can be manifested in even more complex ways than in L1 development. A possible outcome of experience with an L2 is increasing expertise or proficiency in the second language. But such expertise can be modulated by a multitude of other instrumentations of experience, such as frequency of speaking a particular language, age of onset of L2 learning, context of use, which can take the form of learning setting (naturalistic, instructed) and language setting (L1 vs. L2 monolingual speaking environments vs. bi- or multilingual speaking environments), length of immersion in a specific speaking environment, and so on (for a comprehensive discussion of such variables see Bylund \& Athanasopoulos, 2015).

In addition to the diverse instantiations of experience that the process of L2 learning may entail, the L2 learner already has a rich set of conceptual categories associated with L1 linguistic categories. In an associative learning account, such past knowledge will inevitably interact with new information and is always open to restructuring by new information. This observation, along with the multifaceted instantiations of L2 linguistic experience that are subject to considerable individual differences, means that the degree of strength of the direction of this interaction (L1 to L2, and L2 to L1) will depend on a combination of the individual learner's history, as well as the specific linguistic categories under investigation.

$<\mathrm{B}>$ Grammatical Aspect and Motion Event Construal in L2 Users

Based on the robust evidence that speakers of aspect languages tend to exclude endpoints when describing dynamic goal-oriented stimuli, while speakers of non-aspect 
languages display a tendency instead to focus on the endpoints in their verbal descriptions, a recent study by Bylund and Jarvis (2011) analysed the relation between grammatical aspect and verbal processing of endpoints vs ongoingness in goal-oriented motion events in SpanishSwedish bilinguals. They asked their participants to describe the scenes in Spanish (their L1). Results showed that the bilinguals behaved differently from Spanish monolinguals. First, bilingual speakers produced more endpoints in motion events and simple verb forms than Spanish monolinguals. Second, proficiency in L1, measured through the ability to identify aspectual errors in a grammaticality judgment test, was the best predictor of endpoint encoding: The weaker the bilinguals' command of Spanish aspectual distinctions, the higher his/her endpoint encoding frequencies. This finding provides evidence of a direct link between grammatical aspect and endpoint encoding. Age of onset of L2 acquisition was also a significant factor that negatively correlated with endpoint encoding frequencies in the L1.

Flecken (2011) investigated effects of grammatical aspect on verbal encoding in early bilingual speakers of Dutch (an aspect language) and German (a non-aspect language). Her main question was the extent to which these early and simultaneous bilinguals would encode endpoints in their L1 speech, thus showing an effect of the L2 on the L1. Additionally, eye movements were recorded with eye-tracking equipment before and during the description task in order to study planning and organization of content that was going to be expressed. The results showed that bilinguals displayed patterns of language use that were dissimilar to either monolingual norm. Specifically, these early bilinguals frequently used the progressive form in Dutch, but they also tended to combine progressive aspect with the mention of endpoints, a combination that is not at all frequent in monolingual Dutch speakers. The eyetracking analysis demonstrated that bilinguals also allocated attention differently than the monolingual groups. They tended to look earlier and for longer periods to the action than to the agent of the event, and this correlated with a high frequency of use of progressive forms, 
showing a tight link between frequency of use of specific linguistic features and attention allocation to specific aspects of a dynamic event.

More recently, studies have also attempted to study motion event categorization outside of overt speech production. Bylund and Athanasopoulos (2014) correlated frequency of language use with categorization tendencies in native speakers of IsiXhosa who were multilingual in a number of languages. The researchers repeated the memory version of Athanasopoulos and Bylund's (2013) study, which had shown cognitive differences between English and Swedish speaking populations. IsiXhosa being a non-aspect language, the researchers investigated how the knowledge and use of additional languages with grammatical aspect may influence cognition of endpoint-oriented motion events in these functional multilinguals. Results from a triads matching task showed that multilinguals who often used aspect languages and had greater exposure to English (an aspect language) in primary education were less prone to relying on endpoints when making their categorization decisions. Bylund et al. (2013) additionally examined event categorization in L1 speakers of Afrikaans (a non-aspect language) who had English as an L2. Results from a triads matching task showed that those who used English more often were less prone to relying on the reaching of endpoints while categorizing the motion events, thus exhibiting categorization behaviour closer to that of English speakers.

While the recent studies show a tight link between grammatical aspect and motion event construal, some theoretical frameworks view grammatical aspect as the linguistic realization of a more general cognitive representation, that of boundedness. Specifically, Niemeier and Reif's (2008) pedagogically oriented analysis of grammatical aspect posits that sentences containing progressive aspect such as "Mr. Karabatsos is walking" entail an unbounded construal, since the focus is on the ongoing nature of the action, leaving out the boundaries (i.e., the source and the goal of the action). When no progressive marker is used, 
such as in the sentence "Mr. Karabatsos walked (home/to the bowling lane)", the speaker adopts a bounded construal, because the act is complete and the conceptual viewer can construe the boundaries of the action. This analysis is entirely compatible with Langacker's theoretical framework presented earlier. Immediate viewing frames entail unbounded construals, whereas holistic viewing frames entail bounded construals. The presence or absence of grammatical aspect in a language may serve as a reliable and distinctive cue to the construal of events as bounded or unbounded and thus direct differential attention to event boundaries such as endpoints.

Reinterpreting the findings of Athanasopoulos and Bylund (2013) and subsequent studies (Bylund et al., 2013; Bylund \& Athanasopoulos, 2014) under Niemeier and Reif's (2008) account, native speakers of [+ aspect] languages tend to focus more on the unbounded nature of actions as a basis for categorization than native speakers of [- aspect] languages do, because [ + aspect] languages offer more linguistic cues (such as obligatory morphological marking) to their speakers to construe events as unbounded than [- aspect] languages do. This clarifies what is at stake in an L2 learning context between certain language pairs. If the second language employs grammaticized progressive aspect then it offers an important linguistic cue to construe events as unbounded. If the second language does not obligatorily apply such aspectual means to express ongoingness, then this is a reliable cue to interpret events as bounded (unless some adverbial or other means is used to specifically denote the unbounded nature of the event in non-aspect languages). Learning and using an L2 with different aspectual characteristics than the L1 effectively means learning to adjust the relative weight of boundedness and unboundedness as a basis for categorization. Under this interpretation, going from a non-aspect to an aspect language should have similar consequences for categorization as going from an aspect language to a non-aspect language. 
In the former case, linguistic cues to construe events as unbounded will increase, while in the latter case, such cues are absent, promoting a construal of events as bounded.

Under an associative learning account, the key to such readjustment is cumulative experience with the second language. Indeed, the studies reviewed in the previous section are in line with the idea that the strength of the weightings of the elements that make up conceptual representation of motion events as a basis for categorization will change as a result of individual differences in experience, with experience typically operationalized as frequency of language use and proficiency.

$<B>$ Aims of the Current Study

The current study focuses on native speakers of English who are users of L2 German. The study does not distinguish between 'L2 learners' and 'L2 users' because the students in our sample use the L2 as part of their university degree program and continue to be L2 learners.

German, like Swedish, is a non-aspect language, and previous studies have established that German speakers tend to focus on endpoints in both their verbal descriptions and in their attention allocation as measured by eye-tracking (von Stutterheim et al., 2012). We thus first seek to establish whether these cross-linguistic differences occur in a non-verbal paradigm (Athanasopoulos \& Bylund, 2013); after establishing that they do we compare the behaviour of monolinguals to that of the L2 user group, and investigate the several variables (see Table 1) that may modulate L2 user behaviour in general terms. This leads to our first research question:

RQ1. Does learning an L2 with fewer cues for unboundedness (such as the absence of progressive aspect) shift categorization behaviour towards a bounded construal, 
indexed by an increased preference for motion endpoints as a basis for nonverbal similarity judgements?

We then focus on those L2 users who began learning the L2 sequentially via formal classroom instruction and who have had minimal or no immersion in the L2-speaking environment. We investigate effects of length of L2 exposure in an L1 setting and the relationship between cognitive behaviour with other factors in L2 users with different periods of L2 exposure. Thus, our second research question addresses the following issue:

RQ2. Does length of exposure to a second language with grammatical properties that differ from those of the first affect the likelihood that learners' cognitive dispositions will shift towards those of native speakers of the L2?

$<$ A $>$ METHOD

$<B>$ Participants

The participants were 14 monolingual English-speaking adults who were students in the United Kingdom (UK) at the University of Bangor, 14 monolingual German-speaking adults who were students in Germany ${ }^{1}$ at Otto-von-Guericke University, and 76 native speakers of English who were using L2 German as part of their degree in German language or German literature at a UK university (recruited from Bangor and Chester Universities, henceforth referred to as L2 users). Permission to recruit and advertise the study was granted by the programme leaders of the target courses of study, who also allowed access to students on a group testing basis. Given the lack of a formal measure of general proficiency in the German language that can be administered quickly, we asked our participants to self-assess their German proficiency on a scale of 1 "poor" to 4 "advanced" in six different categories, namely speaking, understanding, writing, reading, grammar, and pronunciation (following previous similar studies like Flecken, 2011, and a large body of L2 acquisition research that 
uses self-ratings in the absence of more formal proficiency measures, see Marian, Blumenfeld, \& Kaushanskaya, 2007, for a review). By means of a biographical background questionnaire, we also recorded the L2 learners' age of L2 acquisition (defined here as the age at which they self-reported first started learning German), the time they had previously spent in a German-speaking country, the amount of exposure to German-speaking media (including printed press, radio, television, and internet, measured in hours per week), the amount of interaction with German native speakers (measured in hours per week), and how much they enjoy learning German (measured on a $15 \mathrm{~cm}$ visual analogue scale, where $0 \mathrm{~cm}$ was 'not enjoyed at all' and $15 \mathrm{~cm}$ was 'enjoyed very much'). Table 1 provides a summary of these details.

TABLE 1

$<$ INSERT TABLE 1 ABOUT HERE $>$

Summary of English L2 German Learners' Details

\begin{tabular}{ll}
\hline Mean self-rated L2 proficiency (range) & $3.09 / 4(1-4)$ \\
\hline Mean L2 acquisition age (range) & $\begin{array}{l}12 \text { years old } \\
(2-50 \text { years old) }\end{array}$ \\
\hline Mean length of stay in German-speaking country (range) & $\begin{array}{l}3 \text { months } \\
(0-27 \text { months })\end{array}$ \\
\hline $\begin{array}{l}\text { Mean German-speaking media exposure in hours per week } \\
\text { (range) }\end{array}$ & $2.31(0-50)$ \\
\hline $\begin{array}{l}\text { Mean hours per week interacting with German native speakers } \\
\text { (range) }\end{array}$ & $8.76(0-80)$ \\
\hline $\begin{array}{l}\text { Degree of enjoyment of learning L2 German on a } 15 \mathrm{~cm} \text { visual } \\
\text { analogue scale (range) }\end{array}$ & $11.79 \mathrm{~cm}(7 \mathrm{~cm}-14 \mathrm{~cm})$ \\
\hline
\end{tabular}

Participants ranged in age from 18-51 years; all came from lower middle class suburban backgrounds. They were given a monetary reward for their participation.

$<\mathrm{B}>$ Materials

We elicited nonverbal similarity judgement data using the memory triads matching task used previously in Athanasopoulos and Bylund (2013). The stimuli consisted of thirtyone video clips from the stimulus pool of the research group of von Stutterheim, Carroll, and 
Schmiedtová (see e.g., Athanasopoulos \& Bylund, 2013; Schmiedtová, Carroll, \& von Stutterheim, 2011), and were used in all permissible combinations to create 19 triads, consisting of a target clip and two alternate clips. The target clip was a scene with an intermediate degree of goal orientation in which there was a visible, possible endpoint for the motion, but its arrival was not overtly shown (for example a person walking towards a cafe). One type of alternate, the so-called [- endpoint] alternate, was a video with a low degree of goal orientation showing an entity moving along a trajectory without an obvious endpoint (for example a person walking along a road). The other type of alternate, the so-called [+ endpoint] alternate, was a video with a high level of goal orientation that contained a moving entity that actually reached an endpoint (for example a person walking into a building). Triads were created (and clips edited where necessary) in such a way as to control for manner and direction of motion and number of agents. All clips were 6 seconds long. The videos had been normed for visual similarity in the study of Athanasopoulos and Bylund (2013), such that the [- endpoint $]$ alternates looked similar to the [+ endpoint $]$ alternates.

\section{$<\mathrm{B}>$ Procedure}

Students were tested after their small group tutorials at the university. They remained in the classroom after the end of their tutorial, where they were introduced to the experimenter. The experimenter then gave task instructions to the group and distributed informed consent forms and biographical questionnaires for students to complete while the projector screen was set up for the experiment. Once all details had been filled in, the main experiment was administered using PowerPoint. Participants were informed that they would see video clips arranged in triads on the computer screen, with clip A appearing first, then clip B, and finally clip X (the target). Participants were instructed to indicate whether they thought clip X was more similar to clip A or more similar to clip B, by circling the relevant 
answer on their participant response sheet. Thirty-eight triads were thus presented in an ABX format, where in a counter-balanced design half of the time the [- endpoint] alternate appeared first (clip A) and half of the time it appeared second (clip B), and vice versa for the [+ endpoint] alternate. All 38 triads were presented in different random orders across groups. The precise sequence of the clips in each triad was as follows: Clip A played, followed by clip B, followed by clip X. Participants were instructed to give their responses only after they had watched clip X in its entirety. Clips played immediately after one another, with no pause in-between. Inter-trial interval was self-paced until all participants within the group had circled their answer, at which point the experimenter initiated the next trial.

\section{$<$ A $<$ RESULTS}

We first examined the overall pattern of results (shown in Table 2) by conducting a One-Way ANOVA with Group (English, German, and L2 users) as the independent variable and the number of times participants matched the target clip (X) with the [+ endpoint] alternate (indicating an endpoint preference) as the dependent variable. This ANOVA showed a significant main effect of Group, $F(2,101)=5.46, p<0.05$. Post-hoc Scheffé tests confirmed that the difference was located between the two monolingual groups, with German monolinguals selecting significantly more [+ endpoint] alternates than English monolinguals, $p<0.05$. The L2 user group did not differ significantly from either monolingual group, a pattern typically observed in bilingual cognition studies (Athanasopoulos, 2011).

$$
<\text { INSERT TABLE } 2 \text { ABOUT HERE }>
$$

TABLE 2

Mean Endpoint Preferences (\%) Across Groups in the Triads Matching Task.

\begin{tabular}{ll}
\hline Groups & Endpoint preference (\%) \\
\hline German monolinguals & $36.62(8.86)$ \\
English users of L2 & $29.16(12.34)$ \\
German & \\
English monolinguals & $22.37(7.57)$ \\
\hline
\end{tabular}


Note. Standard deviations are shown in parentheses.

We then sought to probe further the performance of the L2 users according to their year of German studies at university. To this end, we conducted another One-Way ANOVA, this time dividing the L2 users into three different groups. This yielded 39 first-year students, 17 second-year students, and 20 fourth- year students (we could not get access to third-year students because they study abroad in a German-speaking country). We compared similarity judgements using the same dependent variable as before in these three groups, as well as the two monolingual groups. Group averages are shown in Figure 1. The ANOVA showed a significant main effect of group, $F(4,99)=7.37, p<0.05$. Post-hoc Scheffé tests revealed that the fourth- year L2 users and the German monolinguals selected significantly more [+ endpoint] alternates than the English monolinguals and the first-year L2 users did, $p<0.05$ in all cases. This indicates that significant shifts in L2 users' motion event cognition can come about when going from a [+ aspect] language to a [- aspect] language, as a function of experience of studying for a German language related degree.

$<$ INSERT FIGURE 1 ABOUT HERE $>$

\section{FIGURE 1}

Endpoint Preferences (\%) in the Triads Matching Task Across German and English Monolinguals, and English Users of L2 German Split by Year of German Studies at University

Note. Columns show group means. Error bars show standard error of the mean.

The next step in the analysis probed the linguistic or extra-linguistic factors that modulate the degree of L2 user cognitive shift. Thus, the proportion of all 76 L2 users' [+ endpoint] preferences was entered into a Pearson correlation analysis, along with their year of 
university study, their self-perceived L2 proficiency, mean age of L2 acquisition, mean length of stay in a German-speaking country, mean exposure to German-speaking media, mean hours per week interacting with German native speakers, and mean enjoyment of learning German as an L2. As expected based on the previous parametric tests, proportion of [+ endpoint] preference was significantly correlated with the L2 users' year of university study, $r=.461, p<0.05$. The higher the number of the year of study, the more participants were prone to select the [+ endpoint] alternate. The L2 users' [+ endpoint] preferences also correlated significantly with their self-rated L2 proficiency, $r=.285, p<0.05$. As proficiency advances, so does the tendency to show preference for the [+ endpoint] alternate. The proportion of [+ endpoint] preferences did not correlate significantly with any of the other variables.

We then focused on length of L2 exposure. Because the group of L2 users included individuals who had begun learning German simultaneously or shortly after English in a naturalistic setting (see the age of L2 acquisition variable in Table 1), we targeted those L2 users that began learning German exclusively and continuously through formal instruction in secondary school, high school, college, or university. In addition, we excluded from the subsequent analysis individuals who self-reported that they had lived in a German-speaking country for more than 14 months. Given that previous studies show L2 cognitive effects only after 24 months (Athanasopoulos et al., 2010) or 36 months (Cook et al., 2006) in the L2speaking country, it would be reasonable to assume that this variable would have minimal effect in this sample. These measures together would ensure homogeneity in terms of learning context (primarily instructed), age of onset of bilingualism (late childhood learners), and length of immersion in the L2-speaking country (minimal), and thus isolate the variable of length of L2 exposure as much as possible in order to study its effects on cognition. Sixtythree individuals were thus selected for further analysis. A One-Way ANOVA with Group 
(English and German monolinguals, and L2 users) as the independent variable and proportion of [+ endpoint $]$ preference as the dependent variable yielded a significant main effect of group, $F(2,88)=5.43, p<0.05$, with the difference located, as expected, between the monolingual groups (post-hoc Scheffé $p<0.05$ ). The L2 users did not differ significantly from either monolingual group. Table 3 shows the groups' averages.

\section{$<$ INSERT TABLE 3 ABOUT HERE $>$}

\section{TABLE 3}

Mean Endpoint Preferences (\%) in the Triads Matching Task Across Monolingual Groups and L2 Users who Began Learning German via Formal/Classroom Instruction

\begin{tabular}{ll}
\hline Groups & Endpoint preference (\%) \\
\hline German monolinguals & $36.62(8.86)$ \\
English users of L2 German via formal & $28.66(12.62)$ \\
instruction & \\
English monolinguals & $22.37(7.57)$ \\
\hline
\end{tabular}

Note. Standard deviations are shown in parentheses.

As with the whole group, we also conducted a Pearson's correlation with this group, this time adding the variable of years of L2 exposure. The results showed a significant relationship between year of study and [+ endpoint] preferences, $r=.451, p<0.05$, while the correlation between self-rated proficiency and [+ endpoint] preferences just about reached significance, $r=.247, p=0.05$. None of the other variables correlated significantly with [+ endpoint] preferences.

The findings show clearly that length of L2 exposure alone can bring about changes in event cognition of the type typically observed in other populations of L2 users, that is, cognitive behaviour that is in-between that of their monolingual peers. However, the fact that length of L2 exposure did not correlate significantly with [+ endpoint] preferences suggests that the relationship between the two variables may be non-linear. This would contrast with the relationship between cognitive behaviour and other variables such as proficiency, where across many studies, paradigms, linguistic domains, and different L2 user populations, that relationship has been found to be monotonic linear. To probe further this possibility, we 
evenly divided up the 63 L2 users who began learning German through formal instruction into 3 groups based on the years of L2 exposure. This yielded a group of 'short exposure' L2 users with an average of 3.8 years of exposure ( $S D=2.4$ years), a group of 'medium exposure' L2 users with an average of 7.5 years of exposure $(S D=0.5$ years $)$, and a group of 'long exposure' L2 users with an average of 10.8 years of exposure ( $S D=2.5$ years). As Figure 2 shows, our hypothesis about the non-linear relationship between length of L2 exposure and [+ endpoint] preference was confirmed. Short exposure L2 users appear to shift away from monolingual speakers of their L1, towards the direction of their L2, resembling typical in-between behaviour. Medium exposure L2 users on the other hand appear to revert back to the L1 pattern, showing behaviour that is near-identical with their monolingual L1speaking counterparts. Finally, long exposure L2 users shifted completely towards the pattern of monolingual speakers of the L2. A One-Way ANOVA comparing the 3 groups of L2 users and their monolingual peers showed a significant main effect of Group, $F(4,86)=6.83, p<$ 0.05. Post-hoc Scheffé tests showed that the German monolinguals and the long exposure L2 users selected the [+ endpoint] alternate significantly more frequently than did the English monolinguals and the group of medium exposure L2 users (all $p$ values $<0.05$ ). The short exposure L2 users did not differ significantly from any of the other groups.

\section{FIGURE 2}

Endpoint Preferences (\%) in the Triads Matching Task Across German and English Monolinguals and English Users of L2 German, Differentiated by Length of L2 Exposure

Note. Columns show group means. Error bars show standard error of the mean.

We then sought to discover which factors are related to the cognitive behaviour of each group of L2 users according to length of L2 exposure. Thus, for each group of short, 
medium, and long exposure L2 users we performed correlations with the proportion of [+ endpoint] alternate selection and the variables used in the previous correlation. Interestingly, in the short instruction group, [+ endpoint] preferences did not correlate significantly with any other variable. In the medium instruction group, [+ endpoint] preferences correlated with year of study at university, $r=.386, p<0.05$, while the correlation with self-rated proficiency was marginally significant, $r=.361, p=0.05$. In the long instruction L2 user group, [+ endpoint] preferences correlated significantly with self-rated L2 proficiency, $r=.483, \mathrm{p}<$ 0.05 , and with year of study at university, $r=.454, p<0.05$.

\section{$<$ A $>$ DISCUSSION}

The discussion is divided into two main sections: First, we will focus on the main findings of the study, looking at global and specific patterns of performance, and suggest an interpretation of the documented effects of length of L2 exposure, and the evolution of the relationship between proficiency and event categorization patterns; second, we will discuss pedagogical aspects of cognitive restructuring in L2 learning.

\section{$<\mathrm{B}>$ Cognitive Restructuring and Exposure to a Second Language}

We set out to extend the investigation of motion event cognition to a new population of native speakers (German). Results showed that native speakers of English and German differed systematically in their event categorization patterns, establishing the existence of cognitive differences between English and German-speaking populations in event construal beyond overt language production. These findings are consistent with those of previous studies using the same non-verbal paradigm (Athanasopoulos \& Bylund, 2013; Bylund et al., 2013). 
Our primary goals, however, concern L2 user behaviour. Our findings are the first to show an effect of the second language on cognition in individuals whose L1 has progressive aspect but whose L2 lacks that grammatical feature. Although one might not expect cognitive restructuring in the learning of a language that does not involve the acquisition of a specific grammatical construction linked to said hypothetical cognitive restructuring, we provide a principled account for such an influence by referring to the theoretical assumptions used by Niemeier and Reif's (2008). Specifically, what is being restructured is not grammatical aspect per se, but the construal of boundedness and unboundedness, which in turn affects how motion events are categorized by L2 users. Under this interpretation, construal of events as bounded was an aspect of the L2 that initially lacked saliency for the learners. However, the weight of bounded construal as a basis for categorization changed under the influence of long exposure, as a function of increasing L2 proficiency, and with year of study at university.

It is well known that higher levels of L2 proficiency consistently relate to cognitive restructuring in L2 users in a number of domains, such as grammatical number and object categorization (Athanasopoulos, 2006), grammatical gender (Kurinski \& Sera, 2011), and spatial cognition (Park \& Ziegler, 2014). However, studying the evolution of the relationship between proficiency and motion event categorization in learners with different lengths of L2 exposure is able to reveal key insights into the nature of cognitive restructuring, at least in this and similar samples of learners. Short exposure learners seem to display the typical inbetween behaviour that is characteristic in bilingual cognition studies and that is indicative of a shift from L1 behaviour patterns to L2 behaviour patterns. Medium exposure learners seem to revert back to the L1 pattern, showing resistance to restructuring. Long exposure learners appear to have shifted completely towards the L2 cognitive pattern. We interpret this apparent U-shape of cognitive patterns in groups with different lengths of instruction as a special case of the non-linearity that is characteristic of all developmental processes (Gershkoff-Stowe \& 
Thelen, 2004). Specifically, behavioural regression can be understood as part of the ordinary mechanisms of change, which are characterized by collective dynamics of multiple, contingent processes (Rogers, Rakison, \& McClelland, 2004). In the case of L2 users, such processes include sensitivity to statistical regularities in the input, suppression of pre-potent or routinized categorization schemas, and redirection of attention to a novel event construal (Ameel et al., 2005). Under this interpretation, the state of representation at any given developmental stage always depends on its past history. Therefore regression is not really regression, but reflects a developmental stage in changing behaviour (Gershkoff-Stowe \& Thelen, 2004). New cognitive patterns may emerge as a function of the changing relationships between internal processes (suppression and redirection) and contextual influences (statistical regularities in the input).

How might one explain the non-linear curve in this particular sample of learners? Similar curves can be observed in associative learning that takes place over a long period of time, when a specific construal is consistent with input only in one specific domain, but inconsistent in other domains (Rogers et al., 2004). In such cases, statistical learning will initially result in changing cognitive representations as the system is affected at a general level. This can explain the shift observed in the short instruction group. Later on, however, the system moves from general to more specific instances of the newly learned property as it tries to represent it in as fine-grained a way as possible. Because for the learners in this study the majority of instances that the system encounters promotes unbounded construals - the L2 learners continue to use their L1 and interact with other L1 native speakers-behaviour may revert to reflect L1 conceptual dominance. Over time, the L2 user must learn to defocus unboundedness as a basis for categorization, and activate a bounded schema as a basis for categorization. Finally, when sufficient repeated exposure to bounded events in a specific context (e.g., the L2 German classroom, or studying German at University) occurs, the L2 
user is able to overcome any pre-potent bias and shifts towards the L2 cognitive pattern. Evidence that is compatible with the onset of this process can be observed in the medium exposure learners. Even though group averages do not indicate cognitive restructuring, individual patterns of behaviour (as revealed by correlational analyses) do show a propensity to focus on boundedness as a basis for categorization at higher levels of proficiency and with more years of university study. That is, although performance may seemingly deteriorate, there are long-term changes that indicate overall positive growth, as attested to by the significant correlations in the medium exposure group. These correlations then become stronger and more significant in the long exposure group. Thus the apparent U-shaped curve uncovered in the study is unlikely to indicate loss or regression to a former state of representation.

The shift observed in short exposure learners may be attributed to frequency of input alone at a general level, just like the effects of laboratory training of new categories in linguistic relativity studies. Research shows that it is possible to induce changes in categorical perception and object recognition by training participants to learn new artificial categories (Goldstone \& Barsalou, 1998; Notman, Sowden, \& Özgen, 2005; Özgen \& Davies, 2002). However, such effects are transient and short-lived. In line with Ellis (2008), for input to be internalized it needs to be interpreted meaningfully and in context. We view the emergence of a link between L2 proficiency and categorization behaviour in medium exposure learners as the beginnings of such meaningful associative learning, and the progressive strengthening of this correlation in long exposure learners as true internalization of a novel event construal. Even in category training studies it is not that participants lose or unlearn the categories they were exposed to in the laboratory, but over time the weights of the newly learned categories as categorization cues are not strong enough to suppress individuals' previous, routinized way 
of categorizing. In other words, the problem is not one of loss, but of interference suppression, which is amenable to input frequency effects.

Our findings regarding the shift in long exposure learners contrast with those of Malt and Sloman (2003), who found that advanced L2 users with very similar lengths of exposure as our long exposure group exhibited some discrepancies from native speakers' naming patterns and typicality judgments for household objects. It appears, then, that different domains of experience may afford differential opportunities for restructuring. It is important to note, however, that the developmental trends described and examined here and in Malt and Sloman's (2003) study are not developmental trends in the literal sense because these studies are cross-sectional, and focus on adults, relying on their previous linguistic history and their self-assessments of knowledge of the L2 as variables worth investigating in the context of cognitive restructuring in L2 acquisition (cf. Athanasopoulos, 2011).

Nevertheless the trajectory of cognitive behaviour observed in this study as a function of L2 exposure and the progression of influence of increasing expertise in the L2 (as indicated by self-rated proficiency and year of study at university) conforms to well-known general learning theories, with learners displaying behaviour that is fully consonant with theories that postulate orderly progression underlying apparent U-shaped learning. This progression indicates that the apparent L1-like behaviour in medium exposure learners is not just a reflection of 'unlearning' or of L1 entrenchment, but is rather the surface instantiation of a much more complex reorganization of the cognition of these L2 learners, which provides first evidence for true internalization of L2-derived motion event cognition, consistent with theories of domain-general U-shaped learning trajectories. Our assumptions in this regard need to be verified in a longitudinal design that spans several years (cf. Stam, 2015), but the current findings lay a useful methodological and theoretical foundation for such future inquiry. 
$<\mathrm{B}>$ Placing Cognitive Restructuring Within L2 Pedagogy

As already mentioned, in a Cognitive Linguistic approach language is understood to be another instance of general human cognition, inextricably linked to other cognitive abilities and to the multi-modal nature of memory representation, which contains both verbal and non-verbal experiential conceptual elements. Such a view is very much in line with neuroscientific and clinical approaches to representation, where linguistic categories are seen as constituent elements of larger complexes of knowledge (Humphreys \& Forde, 2001; Pulvermüller, 2003). As Tyler (2012) puts ist "language is understood as part and parcel of general cognitive organization and processes" (p. 19).

Is cognitive restructuring of the type investigated and demonstrated in this study an essential part of L2 learning? If verbal communication is part of humans' general cognitive make-up and is constrained and constructed from the same building blocks as other cognitive processes, then the answer is 'yes' and cognitive restructuring in SLA is just as important an outcome of L2 learning as any other aspect of linguistic restructuring, such as phonetic, lexical, grammatical, or pragmatic restructuring. Beyond that, we suggest that a more nuanced and also more principled treatment of the phenomenon might be attained through the notion of 'cognitive' or 'behavioural' accent, here exemplified by endpoint saliency as a function of the presence or absence of progressive aspect in a language. Scholars looking at information structure in L2 users have already introduced the notion of 'semantic accent' to refer to L2 user communication that may in all respects be target-like with regard to grammar and vocabulary, but may nonetheless still be perceived as non-native because of the way L2 users choose to segment information into grammatical and lexical units (Tyler, 2012). Thus, if a German L2 user of English produces sentences containing progressive aspect while consistently mentioning the endpoint (e.g., 'I was driving to the petrol station when John 
rang') even in contexts where the endpoint is irrelevant to the current conversation schema, then an otherwise grammatical utterance may be perceived as having a foreign semantic accent because it presents additional information that a native speaker would not include.

Attaching salience to endpoints is not confined only to overt language use, but to a number of non-verbal activities as well, such as driving, walking, running, cycling, as well as interpreting and remembering acts of motion and the constituent elements of such motion, such as the agent, trajectory, manner, and endpoint. In other words, construal of a motion event is a multifaceted phenomenon that manifests itself in overt verbal behaviour, as well as an array of instances of non-verbal behaviour, which may be verbally mediated to different degrees. In the case of the German L2 English user described earlier, attaching undue importance to the possible endpoint of some action that she herself is performing, or some action that she is recalling from memory in an eye-witness account, may be perceived as displaying a foreign cognitive or behavioural accent. While it may be very difficult for L2 learners to notice, let alone modulate, their L1 semantic accents (since L2 teaching rarely focuses on such aspects of language use), it may be even more difficult for L2 learners to notice and try to overcome a behavioural or cognitive L1 accent. Living in an L2 setting and frequently using the L2 in that setting may help (Bylund \& Athanasopoulos, 2014; Bylund et al., 2013), but the task seems a particularly arduous challenge in an instructed L2 learning setting in the L1-speaking country, for several reasons. First, new information conflicts with fully developed cognitive routines. If humans learn new information more easily and with less effort when they can relate it to established cognitive schemas (Rummelhart, 198ㄴ1), then learning novel or different categorical distinctions in the L2 may pose a particular challenge. Second, frequency of usage of the L2 (which is an important variable in cognitive restructuring occurring in immersed or multilingual settings) tends to be limited to a few hours per week in a classroom, possibly augmented by individual interactive self-study 
activities. As demonstrated in this study, this may have consequences for the internalization of novel construals and shifting categorizations.

Finally, rich opportunities for learners to interact and internalize the behaviour of native speakers of the L2 tend to be confined to second-hand exposure via media interactive teaching materials, or via interaction with the teacher, in cases where the teacher is a native speaker of the L2. Absent other native speakers in the classroom, even in those cases the teacher's behaviour may not be typical of native speakers. Learners rarely, if ever, receive explicit instruction on information structure, and are even less likely to be made aware of a non-verbal 'cognitive' or 'behavioural' accent. For the L2 learner, a behavioural L1 accent would not necessarily cause communication breakdown and, indeed, may not even be relevant in cases where the learner has not lived or does not intend to live in the L2-speaking community. However, if the primary goal of all cognitive activities (including language) is mental contact with other humans (Langacker,1987, 1991), then being aware of and being able to modulate one's cognitive accent becomes one of the central processes of being a bi/multilingual individual, and as such one of the major goals of additional language learning.

What is perhaps needed at the moment to facilitate cognitive restructuring in instructed L2 learners is the fundamental role of interaction between the expert and the novice, as initially conceptualized by Vygotsky (1934/1986) and later filtered through the sensibilities of L2 teaching researchers (e.g., Lantolf, 2003; Lantolf \& Appel, 1994). Under this account, cultural knowers (i.e., teachers or native speakers of the L2) act as models for novices (i.e., L2 learners), who through apprenticeship learning develop the basic cognitive tools and skills needed to accomplish a particular activity, in this case, L2-based cognitive behaviour. Unless such tools and skills are built into a long-term L2 curriculum (see Byrnes et al., 2010; Cook, 2011; Pavlenko, 2009 for some preliminary discussions), cognitive restructuring will remain a significant challenge for the L2 classroom learner. 


\section{NOTES}

${ }^{1}$ Given that it is virtually impossible to find completely monolingual German native speakers who are also educated to University level, we made sure that these participants were not using English as part of their degree; we also administered the Quick Oxford Placement Test, a quick English proficiency test. The mean score on the test was $47.86 \%(S D=8.92)$, indicating that most participants had basic to lower intermediate knowledge of English.

\section{REFERENCES}

Ameel, E., Storms, G., Malt, B. C., \& Sloman, S. A. (2005). How bilinguals solve the naming problem. Journal of Memory and Language, 53, 60-80.

Athanasopoulos, P. (2006). Effects of the grammatical representation of number on cognition in bilinguals. Bilingualism: Language and Cognition, 9, 89-96.

Athanasopoulos. P., \& Kasai, C. (2008). Language and thought in bilinguals: The case of grammatical number and nonverbal classification preferences. Applied Psycholinguistics, 29, 105-123.

Athanasopoulos, P., Dering, B., Wiggett, A., Kuipers, J., \& Thierry, G. (2010). Perceptual shift in bilingualism: Brain potentials reveal plasticity in pre-attentive colour perception. Cognition, 116, 437-443.

Athanasopoulos, P. (2011). Cognitive restructuring in bilingualism. In A. Pavlenko (Ed.), Thinking and speaking in two languages (pp. 29-65). Bristol, UK: Multilingual Matters.

Athanasopoulos, P., \& Bylund, E. (2013). Does grammatical aspect affect motion event cognition? A cross-linguistic comparison of English and Swedish speakers. Cognitive Science, 37, 286-309. 
Baddeley, A. (2003). Working memory: looking back and looking forward. Nature Reviews Neuroscience, 4, 829-839.

Barner, D., Inagaki, S., \& Li, P. (2009). Language, thought, and real nouns. Cognition, $111,329-344$.

Boroditsky, L. (2001). Does language shape thought? English and Mandarin speakers' conceptions of time. Cognitive Psychology, 43, 1-22.

Boroditsky, L., Schmidt, L., \& Phillips, W. (2003). Sex, syntax, and semantics. In D. Gentner \& S. Goldin-Meadow (Eds.), Language in mind: Advances in the study of language and thought (pp. 61-80). Cambridge, MA: MIT Press.

Boutonnet, B., Athanasopoulos, P., \& Thierry, G. (2012). Unconscious effects of grammatical gender during object categorisation. Brain Research, 1479, 72-79.

Bybee, J. (2006). Frequency of use and the organization of language. Oxford: Oxford University Press.

Bylund, E. (2011). Language-specific patterns in event conceptualization: Insights from bilingualism. In A. Pavlenko (Ed.), Thinking and speaking in two languages (pp. 108142). Bristol, UK: Multilingual Matters.

Bylund, E., \& Jarvis, S. (2011). L2 effects on L1 event conceptualization. Bilingualism: Language and Cognition, 14, 47-59.

Bylund, E., Athanasopoulos, P., \& Oostendorp, M. (2013). Motion event cognition and grammatical aspect: Evidence from Afrikaans. Linguistics, 51, 929-955.

Bylund, E., \& Athanasopoulos, P. (2014). Language and thought in a multilingual context: The case of isiXhosa. Bilingualism: Language and Cognition, 17, 431-441.

Bylund, E., \& Athanasopoulos, P. (2015). Linguistic relativity in SLA: Towards a new research programme. Language Learning. 
Bylund, E., \& Athanasopoulos, P. (2015). Televised Whorf: Cognitive restructuring in advanced foreign language learners as a function of audio-visual media exposure. Modern Language Journal, 99-s.

Byrnes, H., Maxim, H. H., \& Norris, J. M. (2010). Realizing advanced L2 writing development in a collegiate curriculum: Curricular design, pedagogy, assessment. Modern Language Journal, 94-s,iv-vi, 1-235.

Casasanto, D. (2008). Who's afraid of the Big Bad Whorf? Cross-linguistic differences in temporal language and thought. Language Learning, 58, 63-79.

Colunga, E., \& Smith, L. B. (2005). From the lexicon to expectations about kinds: A role for associative learning. Psychological Review, 112, 347-382.

Cook, V. J., Bassetti, B., Kasai, C., Sasaki, M., \& Takahashi, J.A. (2006). Do bilinguals have different concepts? The case of shape and material in Japanese L2 users of English. International Journal of Bilingualism, 10, 137-152.

Cook, V. J. (2011). Linguistic relativity and language teaching. In V. J. Cook \& B. Bassetti (Eds.), Language and bilingual cognition (pp. 509-518). Hove, UK: Psychology Press.

Ellis, N. C. (1998). Emergentism, connectionism and language learning. Language Learning, $48,631-664$.

Ellis, N. C. (2002). Frequency effects in language processing: A review with implications for theories of implicit and explicit language acquisition. Studies in Second Language Acquisition, 24, 143-188.

Ellis, N. C. (2006). Selective attention and transfer phenomena in L2 acquisition: Contingency, cue competition, salience, interference, overshadowing, blocking, and perceptual learning. Applied Linguistics, 27, 164-194. 
Ellis, N. C. (2008). Usage-based and form-focused language acquisition: The associative learning of constructions, learned attention and the limited L2 end state. In P. Robinson \& N. C. Ellis (Eds.), Handbook of cognitive linguistics and second language acquisition (pp. 372-406). London: Routledge.

Ellis, N. C., \& Ferreira-Junior, F. (2009). Constructions and their acquisition: Islands and the distinctiveness of their occupancy. Annual Review of Cognitive Linguistics, 7, 188221.

Evans, V., Bergen, B. K., \& Zinken, J. (2007). Cognitive linguistic reader. London: Equinox. Flecken, M. (2011). Event conceptualization by early Dutch-German bilinguals: Insights from linguistic and eye-tracking data. Bilingualism: Language and Cognition, 14, 6177.

Franklin, A., Drivonikou, G. V., Clifford, A., Kay, P., Regier, T., \& Davies, I. R. L. (2008). Lateralization of categorical perception of color changes with color term acquisition. Proceedings of the National Academy of Sciences, 105, 18221-18225.

Geeraertz, D., \& Cuyckens, H. (Eds.) (2007). The Oxford handbook of cognitive linguistics. Oxford: Oxford University Press.

Gershkoff-Stowe, L., \& Thelen, E. (2004). U-shaped changes in behaviour: A dynamic systems perspective. Journal of Cognition and Development, 5, 11-36.

Goldstone, R. L., \& Barsalou, L. W. (1998). Reuniting conception and perception. Cognition, $65,231-262$.

Gopnik, A., Choi, S., \& Baumberger, T. (1996). Cross-linguistic differences in early semantic and cognitive development. Cognitive Development, 11, 197-227.

Harnad, S. (1987). Categorical perception: The groundwork of cognition. Cambridge: Cambridge University Press. 
Harnad, S. (2005). To cognize is to categorize. Cognition is categorization. In H. Cohen \& C. Lefebvre (Eds.), Handbook of categorization in cognitive science (pp . 20-45). London: Elsevier.

Humphreys, G. W., \& Forde, E. M. E. (2001). Hierarchies, similarity, and interactivity in object recognition: "Category-specific" neuropsychological deficits. Behavioral and Brain Sciences, 24, 453-509.

Imai, M., \& Gentner, D. (1997). A crosslinguistic study of early word meaning: Universal ontology and linguistic influence. Cognition, 62, 169-200.

January, D., \& Kako, E. (2007). Re-evaluating evidence for linguistic relativity: Reply to Boroditsky (2001). Cognition, 104, 417-426.

Jarvis, S., \& Pavlenko, A. (2008). Crosslinguistic influence in language and cognition. New York: Routledge.

Johnson, E. K., \& Jusczyk, P. W. (2001). Word segmentation by 8-month-olds: When speech cues count for more than statistics. Journal of Memory and Language, 44, 548-567.

Kersten, A., Meissner, C., Lechuga, J., Schwartz, B. , Albrechtsen, J., \& Iglesias, A. (2010). English speakers attend more strongly than Spanish speakers to manner of motion when classifying novel objects and events. Journal of Experimental Psychology: General, $139,638-653$.

Kiefer, M., \& Pulvermüller, F. (2012). Conceptual representations in mind and brain: Theoretical developments, current evidence and future directions. Cortex, 48, 805825.

Kurinski, E., \& Sera, M. D. (2011). Does learning Spanish grammatical gender change English-speaking adults' categorization of inanimate objects? Bilingualism: Language and Cognition, 14, 203-220. 
Langacker, R. (1987). Foundations of cognitive grammar. Vol 1. Stanford, CA: Stanford University Press.

Langacker, R. (1991). Foundations of cognitive grammar. Vol 2. Stanford, CA: Stanford University Press.

Langacker, R. (2008). Cognitive grammar: A basic introduction. New York: Oxford University Press.

Lantolf, J. P., \& Appel, G. (Eds.) (1994). Vygotskian approaches to second language research. Norwood, NJ: Ablex.

Lantolf, J. P. (2003). Intrapersonal communication and internalization in the second language classroom. In A. Kozulin, V. S. Ageev, S. Miller, \& B. Gindis (Eds.), Vygotsky's educational theory in cultural context. (pp. 349-370). Cambridge: Cambridge University Press.

Li, P., \& Gleitman, L. (2002). Turning the tables: Language and spatial reasoning. Cognition, $83,265-294$.

Lucy, J. A. (1992). Grammatical categories and cognition: A case study of the linguistic relativity hypothesis. Cambridge: Cambridge University Press.

Lucy, J. A. (1997). Linguistic relativity. Annual Review of Anthropology, 26, 291-312.

Lucy, J. A., \& Gaskins, S. (2001). Grammatical categories and the development of classification preferences: A comparative approach. In M. Bowerman \& S. Levinson (Eds.), Language acquisition and conceptual development (pp. 257-283). New York: Cambridge University Press.

Lucy, J. A., \& Gaskins, S. (2003). Interaction of language type and referent type in the development of nonverbal classification preferences. In D. Gentner \& S. GoldinMeadow (Eds.), Language in mind: Advances in the study of language and thought (pp. 465-492). Cambridge, MA: MIT Press. 
Majid, A., Bowerman, M., Kita, S., Haun, D. B. M., \& Levinson, S. C. (2004). Can language restructure cognition? The case for space. Trends in Cognitive Sciences, 8, 108-114.

Malt, B. C., \& Sloman, S. (2003). Linguistic diversity and object naming by non-native speakers of English. Bilingualism: Language and Cognition, 6, 47-67.

Marian, V., Blumenfeld, H. K., \& Kaushanskaya, M. (2007). The Language Experience and Proficiency Questionnaire (LEAP-Q): Assessing language profiles in bilinguals and multilinguals. Journal of Speech, Language, and Hearing Research, 50, 940-967.

Niemeier, S., \& Reif, S. (2008). Making progress simpler? Applying cognitive grammar to tense-aspect teaching in the German EFL classroom. In S. De Knop \& T. De Rycker (Eds.), Cognitive approaches to pedagogical grammar (pp. 325-356). Berlin/New York: Mouton de Gruyter.

\section{Nosofsky, R. M. (1986). Attention, similarity, and the identification-categorisation}

\section{relationship. Journal of Experimental Psychology: General, 115, 39-57.}

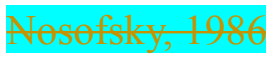

Notman, L., Sowden, P. T., \& Özgen, E. (2005). The nature of learned categorical perception effects: A psychophysical approach. Cognition, 95, B1-B14.

Özgen, E., \& Davies, I. R. L. (2002). Acquisition of categorical color perception: A perceptual learning approach to the linguistic relativity hypothesis. Journal of Experimental Psychology: General, 131, 477-493.

Park, H. I., \& Ziegler, N. (2014). Cognitive shift in the bilingual mind: Spatial concepts in Korean-English bilinguals. Bilingualism: Language and Cognition, 17, 410-430.

Pavlenko, A. (2009). Conceptual representation in the bilingual lexicon and second language vocabulary learning. In A. Pavlenko (Ed.), The bilingual mental lexicon: Interdisciplinary approaches (pp. 125-160). Clevedon, UK: Multilingual Matters.

Pavlenko, A. (Ed.). (2011). Thinking and speaking in two languages. Bristol, UK: Multilingual Matters. 
Pulvermüller, F. (2003). The neuroscience of language: On brain circuits of words and serial order. Cambridge: Cambridge University Press.

Regier, T., \& Kay, P. (2009). Language, thought and color: Whorf was half right. Trends in Cognitive Sciences, 13, 439-446.

Roberson, D., Davidoff, J., Davies, I., \& Shapiro, L. (2004). The development of color categories in two languages: A longitudinal study. Journal of Experimental Psychology: General, 133, 554-571.

Roberson, D. (2005). Color categories are culturally diverse in cognition as well as in language. Cross-Cultural Research, 39, 56-71.

Rogers, T. T., Rakison, D. H., \& McClelland, J. L. (2004). U-shaped curves in development: A PDP approach. Journal of Cognition and Development, 5, 137145.

Rumelhart, D. E. (1984). Schemata and the cognitive system. In R. Wyer \& T.K. Thomas (Eds.), Handbook of social cognition, Vol 1 (pp. 161-188). Mahwah, NJ, US: Lawrence Erlbaum Associates.

Samuelson, L. K. (2002). Statistical regularities in vocabulary guide language acquisition in connectionist models and 15- to 20-month-olds. Developmental Psychology, 38, 1016-1037.

Schmiedtová, B., \& Flecken, M. (2008). Aspectual concepts across languages: Some considerations for second language learning. In S. de Knop (Ed.), Pedagogical grammar (pp. 357-384). Berlin: Mouton de Gruyter.

Schmiedtová, B., Carroll, M., \& Stutterheim, C. von (2011). Implications of languagespecific patterns of event construal of advanced L2 speakers. In A. Pavlenko (Ed.), Thinking and speaking in two languages (pp. 66-107). Bristol, UK: Multilingual Matters. 
Smith, L. B. (2000). Learning how to learn words: An associative crane. In R. Golinkoff \& K. Hirsh-Pasek et al. (Eds.), Becoming a word learner (pp. 51-80). New York: Oxford University Press.

Smith, L. B. (2003). Learning to recognize objects. Psychological Science, 14, 244-250.

Stam, G. (2015). Changes in thinking for speaking: a longitudinal case study. Modern Language Journal, 99-s.

Stutterheim, C. von (1997). Zum Ausdruck von Raum- und Zeitkonzepten in deutschen und englischen Texten [The expression of spatial and temporal concepts in German and English texts]. Zeitschrift für Germanistische Linguistik, 25, 147-166.

Stutterheim, C. von (2003). Linguistic structure and information organisation. The case of very advanced learners. EuroSLA Yearbook, 3, 183-206.

Stutterheim, C. von, Andermann, M., Carroll, M., Flecken, M., \& Schmiedtová, B. (2012). How grammaticized concepts shape event conceptualization in language production: Insights from linguistic analysis, eye tracking data, and memory performance. Linguistics, 50, 833-867.

Szagun, G. (2001). Learning different regularities: The acquisition of noun plurals by German-speaking children. First Language, 21, 109-141.

Tyler, A. (2012). Cognitive linguistics and second language learning. London: Routledge. Vygotsky, L. S. (1934/1986). Thought and language. Cambridge, MA: MIT Press.

Whorf, B. L. (1956). Language, thought, and reality. Selected writings of Benjamin Lee Whorf (ed. by J. B. Carroll). Cambridge, MA: MIT Press.

Wolff, P., \& Holmes, K. J. (2010). Linguistic relativity. Wiley Interdisciplinary Reviews: Cognitive Science, 2, 253-265. 
